\title{
Feasibility study into a computational approach for marine propeller noise and cavitation modelling
}

\author{
Artur K. Lidtke ${ }^{\mathrm{a}, *}$, Victor F. Humphrey ${ }^{\mathrm{b}}$, Stephen R. Turnock ${ }^{\mathrm{a}}$ \\ ${ }^{a}$ Fluid Structure Interactions Group, University of Southampton, SO17 1BJ, UK \\ ${ }^{b}$ Institute of Sound and Vibration Research, University of Southampton, SO17 1BJ, UK
}

\begin{abstract}
There is increased interest in the ability to predict the noise associated with commercial ship propellers. Key components of the computational analysis process are considered for two test cases and the future direction in resolving the associated challenges is presented. Firstly, the Potsdam Propeller Test Case is used to compute tonal blade passage noise using the Ffowcs Williams-Hawkings acoustic analogy. Cavitation extents predicted using the Sauer and Schnerr mass transfer model agree well with the experiment but show little unsteadiness due to URANS being used. A complementary study of initial results from the study of cavitation noise modelling attempt are presented for a NACA0009 section, used as a simplified representation of a propeller blade. Large Eddy Simulation and FW-H acoustic analogy are used in order to estimate the cavitation-induced noise. Results indicate that the discussed approach provides the means for identifying low-frequency noise generation mechanisms in the flow, but does not allow for the fine-scale bubble dynamics or shockwave formation to be resolved. It is concluded that the discussed approach is a viable option to predict large parts of the marine propeller noise spectra but still further work is needed in order to account for the broadband components.
\end{abstract}

Keywords: Cavitation, Noise, Marine Propeller, Hydrofoil, Acoustic analogy

\section{Introduction}

Concerns about limiting the input of noise into the Oceans have been increasingly more pronounced in recent years. One may associate the anthropogenic noise with multiple mechanisms, shipping being one of the larger contributors (Hildebrand, 2009, Urick 1984). The significance of this is even greater given that a large part of the energy of the ship-related noise falls within the $10-1000 \mathrm{~Hz}$ regime and thus has a high potential to effect marine wildlife (Lloyd 2014).

Hence, several initiatives have been established in order to investigate how to mitigate the impact of shipping on the marine environment (Van der Graaf et al. 2012, Tasker et al. 2010). These have contributed to the debate as to whether regulation should be introduced and updated where necessary in order to limit the noise induced by commercial vessels (Kellet et al. 2014, Bertschneider et al. 2014). According to the review by the ITTC Specialist Committee on Hydrody-

\footnotetext{
* Corresponding author.

Email address: akl1g09@soton.ac.uk (Artur K. Lidtke )
}

namic Noise (Bertschneider et al. 2014) the noise due to the vibration of the hull structure induced by its interaction with the propeller and ship board machinery will be of smaller interest as far as marine wildlife is concerned, although it is of key importance for the crew and passenger comfort on board a commercial ship. It may, however, play a role if the dominant machinery frequencies, such as engine rpm, will coincide with frequency range of particular importance to a given species. This may be overcome by increasing vibration impedance of the structure, for example by avoiding rigidly mounted engines.

The tonal noise sources associated with the propeller, cavitating and non-cavitating, are typically considered to be dominant when assessing the environmental impact of shipborne noise. This is because of their high sound power and low attenuation resulting in the potential to affect the largest area most severely (Bertschneider et al., 2014). It is likely, however, that other noise sources, such as those due to machinery or broadband cavitation, will become of greater importance in offdesign conditions, such as when operating in shallow coastal waters, during manoeuvring or while at port.

January 7, 2016 
The tonal, non-cavitating sound component is caused primarily with the loading noise caused by the blade passing through non-uniform wake of the hull as it rotates (Lloyd et al. 2015, Ianniello et al. 2013). Due to the induced change to the pressure distribution on the blades this phenomenon also has an effect on periodic cavitation. This fluctuation of cavitation volume will act as a strong monopole noise source (Park et al. 2009, Seol et al. 2005, Salvatore \& Ianniello 2002). This may also be expected to be accompanied by contributions from higher order acoustic sources, particularly for smaller source-receiver distances (Seol 2013).

The unsteadiness of the flow will play a crucial role in determining the noise signature of alifting surface such as a propeller or hydrofoil. Thus, while some useful insights may be gained into the cavitation phenomena using approaches such as unsteady RANS or boundary element methods, it is likely that Large Eddy Simulation (LES) will be required to develop a deeper understanding of the underlying flow.

The work reported contributes to a wider study into the assessment of the environmental impact of a ship on marine ecosystems which requires computation of propeller-induced noise levels. Specifically the current focus is to assesses the potential benefits and disadvantages of turbulence and cavitation modelling techniques from the numerical propeller noise modelling perspective.

In order to allow more detailed analysis to be undertaken a basic understanding of the limitations of the modelling methods constituting the current state of the art must be developed. This is done on the example of the Potsdam Propeller Test Case (PPTC). This has seen a significant amount of both experimental and theoretical attention (Abdel-Maksoud 2011), thus becoming one of the more established validation problems. The presented results were obtained using the SchnerrSauer mass transfer cavitation model for the flow being solved using unsteady RANS with the k- $\omega$ SST turbulence model (Sauer \& Schnerr 2001).

The flow over a propeller may be regarded as complex and is thus not well suited for preliminary simulations aimed at assessing the mechanism of cavitation noise. Hence, a simpler test case of a NACA0009 hydrofoil is considered, where LES is used instead of RANS to solve the equations of motion of the flow. The far-field sound pressure level is computed using a porous Ffowcs-Williams Hawking acoustic analogy implemented in OpenFOAM. The presented analysis focuses on correlating the relationships between the predicted flow features and the corresponding noise signals, allowing for preliminary conclusions to be drawn with respect to the aptness of the presented approach to the modelling of noise of a complete propeller.

\section{Numerical modelling}

\subsection{Cavitation}

Cavitation may be described as the transition of liquid into vapour in regions of low pressure. This is caused by the presence of small gas nuclei in the liquid (Plesset \& Prosperetti, 1977). When subject to tensile stress, these nuclei expand and lead to different types of cavitation, such as sheet or bubble cavitation, depending on the flow conditions (Vallier, 2013).

It is possible to simulate the behaviour of individual cavitation bubbles, as described, for instance, by Jamaluddin et al. (2011) and Hsiao \& Chahine (2004). However, because of the small size of the cavitation nuclei, ranging between 2 and $50 \mu \mathrm{m}$ for standard sea water (Woo Shin 2010), it would not be feasible to compute the behaviour of every individual bubble in full detail for a flow over a full-scale propeller or a hydrofoil.

Alternatives involve, for instance, the use of volumeof-fluid or level-set multi-phase flow solvers in order to describe the physics governing the motion of large cavities. Schnerr-Sauer cavitation model has been used here in order to account for the pressure-induced phase change of liquid into vapour and vice versa (Sauer \& Schnerr 2001). This is done based on solving the transport equation for avolume fraction, $\alpha$, with an additional source term introduced on the right-hand side to account for the evaporation and condensation:

$$
\frac{\partial \alpha}{\partial t}+\nabla \cdot(\alpha \mathbf{U})=-\frac{\dot{m}}{\rho},
$$

where $\dot{m}$ denotes the rate of change of mass of the liquid-vapour mixture, $\rho$ is the density of the mixture and $\mathbf{U}$ is the fluid velocity. The presence of the additional source term also modifies the continuity equation which now becomes

$$
\nabla \cdot \overline{\mathbf{U}}=\left(\frac{1}{\rho_{v}}-\frac{1}{\rho_{l}}\right) \dot{m},
$$

where subscripts $v$ and $l$ refer to vapour and liquid phases, respectively. One may also define the density and viscosity of the liquid-vapour mixture as

$$
\begin{aligned}
& \rho=\alpha \rho_{v}+(1-\alpha) \rho_{l}, \\
& \mu=\alpha \mu_{v}+(1-\alpha) \mu_{l},
\end{aligned}
$$

respectively. 
In order to close the system of equations, an expression for the rate of mass transfer between the liquid and the vapour has to be introduced. In the approach proposed by Sauer and Schnerr this is done by considering the equation of motion of a single bubble and rearranging it as

$$
\dot{m}=\frac{\rho_{1} \rho_{\mathrm{v}}}{\rho}(1-\alpha) \alpha \frac{3}{R} \sqrt{\frac{\frac{2}{3}\left(p-p_{\mathrm{v}}\right)}{\rho_{\mathrm{l}}}},
$$

where $R$ is modelled based on the specified characteristic nuclei radius, $R_{0}$, and their volumetric density, $n_{0}$.

\subsection{Large Eddy Simulation}

In the discussed hydrofoil study Large Eddy Simulation (LES) was used in order to model the fluid flow. Use was made of the implicit PISO solver on a collocated finite-volume grid, as implemented in OpenFOAM 2.2.2. The LES approach is based on resolving the most prominent turbulent structures and modelling the remainder of the turbulent kinetic energy spectrum. This is achieved by filtering the momentum equation yielding

$$
\frac{\partial \overline{\mathbf{U}}}{\partial t}+\nabla \cdot(\overline{\mathbf{U}} \otimes \overline{\mathbf{U}})=-\frac{1}{\rho} \nabla \bar{p}+v \nabla^{2} \overline{\mathbf{U}}-\nabla \cdot \tau,
$$

where the overbar denotes the filtering operation, $p$ is the fluid pressure and $v$ is the kinematic viscosity. Similarly, the continuity equation becomes

$$
\nabla \cdot \overline{\mathbf{U}}=0 .
$$

the non-linear subgrid stress tensor, $\tau$, used to describe the effect of the filtered eddies on the flow in Equation(5), may be expressed as

$$
\boldsymbol{\tau}=\overline{\mathbf{U} \otimes \mathbf{U}}-\overline{\mathbf{U}} \otimes \overline{\mathbf{U}} .
$$

In order to model this quantity one may consider the Boussinesq hypothesis, whereby the stress tensor is assumed proportional to the fluid strain-rate and an assumed subgrid viscosity, $v_{S G S}$, yielding

$$
\boldsymbol{\tau}-\frac{1}{3} \boldsymbol{\tau} \cdot \mathbf{I}=2 v_{S G S} \mathbf{S}
$$

In the above $\mathbf{I}$ is the identity matrix, and the strain rate may be computed as

$$
\mathbf{S}=\frac{1}{2}\left(\nabla \mathbf{U}+\nabla \mathbf{U}^{T}\right)
$$

An expression provided by the Smagorinsky model assumes the subgrid scale viscosity to be dependent on a constant coefficient, $C_{S}$, and the filter width, $\Delta$, dictated by the mesh density. These yield an expression:

$$
v_{S G S}=\left(C_{S} \Delta\right)^{2}|\mathbf{S}| \text {. }
$$

\subsection{Ffowcs Williams-Hawkings Acoustic Analogy}

Ffowcs-Williams Hawkings (FWH) acoustic analogy uses the extended Lighthill's equation to predict noise due to turbulence (Ffowcs Williams \& Hawkings, 1969). It does so by transforming noise sources present inside a volume of fluid into a set of surface and a single volume integral using the Green's function. If the integrals are evaluated on a control surface surrounding a region of fluid the so-called porous formulation, formally derived by Di Francescantonio (1997), is used. A key concept in using this method is that no additional modelling of the noise sources needs to be carried out, as long as their effect on the flow within the integration surface is correctly accounted for. In the context of the present work this would imply that as cavitation is created and varies in volume it must displace the flow in accordance with the momentum and mass conservation equations, for instance.

Based on rearranging the mass and momentum conservation equations of the fluids the acoustic analogy introduces a solution to the inhomogeneous wave equation of the form

$$
p^{\prime}(\mathbf{x}, t)=p_{T}^{\prime}(\mathbf{x}, t)+p_{L}^{\prime}(\mathbf{x}, t)+p_{Q}^{\prime}(\mathbf{x}, t)
$$

where $\mathbf{x}$ and $t$ are the receiver position and time, respectively, $p^{\prime}$ is the acoustic pressure disturbance, and subscripts T, L and Q refer to the thickness, loading and non-linear contributions (Lyrintzis, 2002, Ianniello et al. 2012). Each of the terms on the right-hand-side of Equation (11) is computed by evaluating a surface integral of quantities dependent on the state of the flow.

In the case of the porous formulation the non-linear term for sources located within the control surface are accounted for via the thickness and loading contributions. It should be noted that in this case $p_{Q}^{\prime}(\mathbf{x}, t)$ tends to zero only in a strictly numerical sense. This assumption may not hold true if there are noise sources present outside or leaving the control surface. Similarly, for such a formulation the monopole and dipole contributions lose their physical meaning (Ianniello et al. 2012). It is important to note that the quadrupole noise source outside of the control surface is described by a volume integral, which makes it relatively expensive to compute if high sampling frequencies are desired. Instead, it may thus be more convenient to design the porous data surface in such a way as to enclose as many of the relevant noise sources as possible. In practice this may prove difficult, particularly for marine propeller cases which exhibit a well-defined wake with strong vortical structures over a large distance downstream. 
FW-H analogy makes use of two intermediate variables, $U_{i}$ and $L_{i}$, which, for a stationary control surface, may be written as

$$
\begin{aligned}
U_{i} & =\frac{\rho}{\rho_{0}} u_{i}, \\
L_{i} & =P_{i j} \hat{n}_{j}+\rho u_{i}\left(u_{i} \cdot \hat{n}_{i}\right),
\end{aligned}
$$

where $u$ is the flow velocity and $P_{i j}$ is the compressive stress tensor. Given the pressure field of a fluid, $p$, and its value when the fluid is at rest, $p_{0}$, one may simplify the $P_{i j}$ tensor to $p-p_{0}$ (Lyrintzis, 2002,Salvatore, 2009). For compressible flow the fluid density is composed of a steady value, $\rho_{0}$ and a density disturbance, $\rho^{\prime}$, yielding $\rho=\rho^{\prime}+\rho_{0}$. In this study an incompressible flow formulation is used and the fluctuating term is neglected.

For the case of a stationary control surface the general form of the terms of the porous FW-H equation may be computed as

$$
\begin{aligned}
& 4 \pi p_{T}^{\prime}(\mathbf{x}, t)=\int_{S}\left[\frac{\rho_{0} \dot{U}_{n}}{r}\right]_{\tau} \mathrm{d} S, \\
& 4 \pi p_{L}^{\prime}(\mathbf{x}, t)=\frac{1}{c_{0}} \int_{S}\left[\frac{\dot{L}_{r}}{r}\right]_{\tau} \mathrm{d} S+\int_{S}\left[\frac{L_{r}}{r^{2}}\right]_{\tau} \mathrm{d} S .
\end{aligned}
$$

Here $c_{0}$ denotes the speed of sound in the medium $r$ is the magnitude of the radiation direction, dot defines a source time derivative, and subscripts $r$ and $n$ refer to the dot product of the quantity in question with a unit vector in either radiation or normal directions, respectively.

In order to account for the fact that the sound contribution of an infinitesimal control surface element will take afinite amount of time to travel between the source and the receiver all of the quantities in Equations (13) must be evaluated at an appropriate emission time, $\tau$, given by

$$
t=\tau+\frac{|\mathbf{x}-\mathbf{y}|}{c},
$$

where $\mathbf{y}$ is the location of the source (integration surface element). In the current implementation of the $\mathrm{FW}-\mathrm{H}$, developed for the purpose of the discussed project, the control surface is defined by a set cell faces. This provides less control over the density and shape of the control surface than if the flow field was interpolated onto an independent discrete surface. On the other hand, the approach used introduces no additional errors and avoids local pressure and velocity perturbations from being lost.

\section{Potsdam Propeller Test Case}

\subsection{Simulation set-up}

The discussed controllable pitch propeller has parameters as described in Table 1. The presented work focuses on replicating the conditions from the experimental test case 2.3.1, where cavitation measurements were made for the propeller at the advance coefficient of 1.019 , rps-based cavitation number of 2.024 and operating at 24.987 revolutions per second (Abdel-Maksoud 2011). An implicit time stepping algorithm with $\delta t=$ $3 \cdot 10^{-6} s$ was used to solve the unsteady flow.

The propeller and shaft were treated as non-slip walls with wall-functions applied, velocity inlet and pressure outlet boundary conditions were used to impose the axial flow, with the outer extents of the domain assumed to be slip walls. To match the experimental conditions the water and vapour were taken to have densities of 997.44 and $0.023 \mathrm{kgm}^{-3}$, and kinematic viscosities of $9.337 \cdot 10^{-7}$ and $4.273 \cdot 10^{-6} \mathrm{kgm}^{-2}$. The saturation pressure was taken to be $2818 \mathrm{~Pa}$.

Table 1: Parameters of the VP1304 (PPTC) propeller.

\begin{tabular}{lll}
\hline Parameter & Unit & Value \\
\hline Diameter & $\mathrm{m}$ & 0.250 \\
Pitch ratio $r / R=0.7$ & - & 1.635 \\
Chord $r / R=0.7$ & $\mathrm{~m}$ & 0.10417 \\
Skew & deg & 18.837 \\
Hub ratio & - & 0.3 \\
Number of blades & - & 5 \\
rps & $s^{-1}$ & 25 \\
\hline
\end{tabular}

An unstructured hexahedral mesh was created using the snappyHexMesh utility native to OpenFOAM 2.2.2 and consisted of 8.8 million cells. Additional refinement was applied around the propeller tips and roots in order to refine the relevant geometry details. Care was taken to ensure that the $y+$ falls within the logarithmic boundary layer region in order for the wall function models to be valid $(y+\epsilon<30,200>)$. A cylindrical domain was considered as it was deemed that modelling the cavitation tunnel walls as present in the experiment would be too expensive computationally. In order to account for the rotation of the propeller a sliding mesh interface was used. An overview of the mesh and domain arrangement is presented in Figure 1.

First order time discretisation was used which was considered appropriate as only the low-frequency features of the flow were of interest. The convection term of the RANS equation being resolved using second order upwind scheme to better resolve the wake of the propeller. First order schemes were used to model the turbulent quantities and van Leer scheme with interface compression was applied to the volume fraction field. 


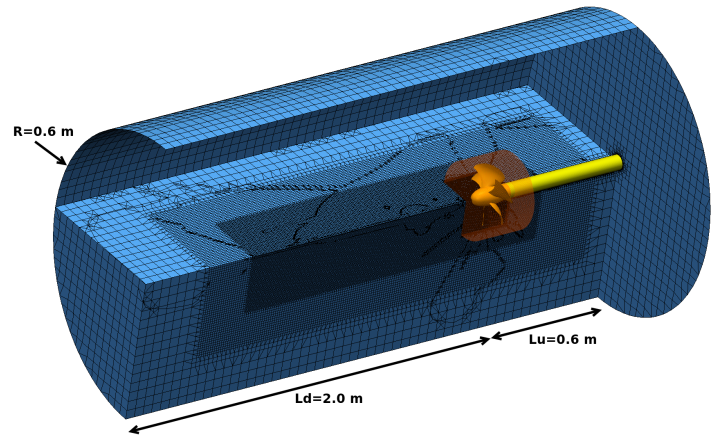

Figure 1: Overview of the domain setup for the PPTC simulation (rotating mesh zone highlighted in orange).

\subsection{Results and Discussion}

One of the primary considerations for this part of the study was analysing how well the selected cavitation model predicts the extents of cavitation for a marine propeller operating close to its maximum efficiency point. As shown in Figure 2, a relatively good agreement may be observed between the predicted and measured location of the cavitation regions. One of the drawbacks, however, is the lack of the tip vortex extending downstream of the propeller This is caused by lack of appropriate refinement of the mesh away from the propeller blade and by the fact that RANS methods in general tend to introduce too much dissipation and thus cause the vortices to disappear much sooner than they would in reality unless appropriate local mesh refinement is applied (Turnock et al., 2006, Phillips \& Turnock., 2013).

Despite the relative coarseness of the mesh an accurate prediction of the thrust coefficient was achieved in non-cavitating conditions, yielding 0.3740 against the experimental value of 0.3870 , i.e. $3.36 \%$ relative error. This indicates that the presented method is well suited to provide information useful throughout the propeller design cycle. Unsteady RANS is not able to predict the unsteady behaviour of the cavities particularly well (Bensow \& Liefvendahl, 2008, Lidtke et al. 2014), nor is it capable of resolving the tip vortex regions accurately. Both of these phenomena may be expected to play a significant role in the noise generation mechanisms of a complete marine propeller (Salvatore, 2009). It is therefore desirable to use Large Eddy Simulation, or similar high-fidelity turbulence modelling techniques, for the purpose of noise prediction.

Due to the simplifications made to the Navier-Stokes equations in the RANS approach the noise due to turbulence could not be reliably predicted in the present

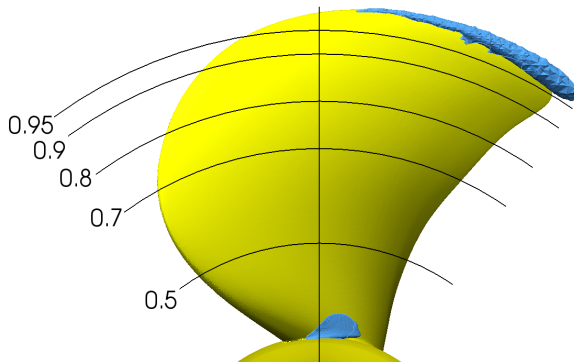

(a) CFD

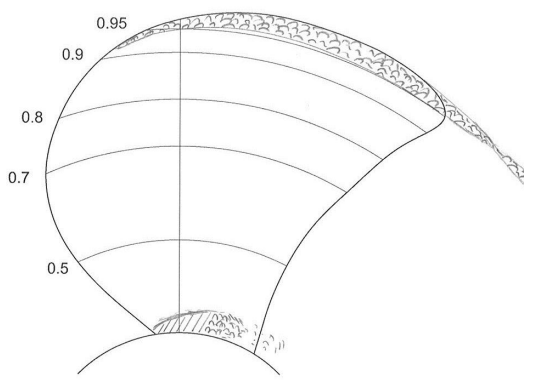

(b) EFD

Figure 2: Comparison of the experimental and computed cavitation extents (experimental data from AbdelMaksoud (2011)). The predicted interface was assumed at volume fraction value of 0.95 .

simulation. In a number of studies, however, it has been reported that the blade-pass frequency components may be predicted well without the use of more complex turbulence modelling techniques (Ianniello et al., 2013, Lloyd et al., 2015). A final part of the PPTC study thus involved using the FW-H acoustic analogy in order to study this part of radiated pressure. A cylindrical porous data surface was defined just inside of the fine mesh region in Figure 1, approximately $5 D$ downstream of the propeller plane and with diameter of $2 D$. Pressure signals were computed for a receiver at the propeller plane and $100 \mathrm{~m}$ away, which corresponds to approximately 8 wavelengths at the BPF.

Figure 3 presents the sound pressure level at the receiver, computed according to

$$
S P L=10 \log \left(\frac{p^{\prime}}{p_{\text {ref }}}\right)^{2},
$$

with the reference pressure level $p_{\text {ref }}$ of $1 \mu \mathrm{Pa}$. One may note that a dominant peak in the spectrum is associated with the blade pass frequency, and is followed by higher order harmonics, as expected. The cavities 
present on the blades have been observed to be stable, thus yielding little volume oscillations. This was the case because of steady, uniform inflow being used and the cavitation number being favourable. Consequently, no additional noise source was predicted due to cavitation other than due to the attached cavitation acting to effectively modify the shape of the propeller blades.

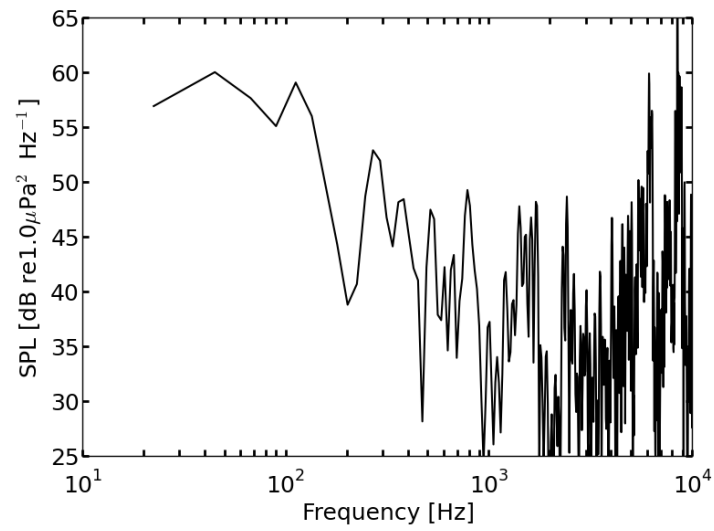

Figure 3: Sound pressure levels computed for the PPTC case at a receiver placed $100 \mathrm{~m}$ away from the shaft centreline at the propeller plane.

\section{NACA0009 Cavitation Noise}

\subsection{Case set-up}

Numerical simulations aimed at providing initial noise estimates of a cavitating hydrofoil have been focused on awing with a NACA0009 section profile. This was done in order to replicate the conditions used for the Delft Twist 11 foil first presented by Foeth et al. (2006). In their study a wing with a span-wise angle of attack variation symmetric about the mid-span was considered. Here, however, the geometry has been simplified to a fixed span-wise pitch distribution in order to allow a more in-depth study of the sheet cavity behaviour without the added complexity dictated by the complex three-dimensional flow features reported in the original experiments. The span of the domain is deemed sufficient to capture the major influences of spanwise fluctuations.

The foil with chord of $0.15 \mathrm{~m}$, angle of attack of $9^{\circ}$ and span of $0.05 \mathrm{~m}$ was placed in the centre of a domain which was to resemble the working section of the cavitation tunnel used by Foeth et al. The domain was chosen to extend 2.5 chord lengths upstream, $4.5 c$ downstream, and was $2.5 c$ wide. The densities of both fluids were taken to be $998 \mathrm{kgm}^{-3}$ and $0.023 \mathrm{kgm}^{-3}$ for water and vapour, respectively, and their corresponding kinematic viscosities were assumed to be $10-6 \mathrm{kgm}^{-2}$ and $4.273 \cdot 10^{-6} \mathrm{kgm}^{-2}$. The mean nucleation radius was assumed to be $50 \mu \mathrm{m}$ with the corresponding distribution of $10^{8} \mathrm{~m}^{-3}$. Finally, the saturated vapour pressure of the mixture was taken to be $2970 \mathrm{~Pa}$. Speed of sound in water was assumed to be $1500 \mathrm{~ms}^{-1}$. As in the propeller case, the implicit PISO algorithm was used to solve the flow at a time step of $2 \cdot 10^{-6} \mathrm{~s}$, guaranteeing maximum $C o \leq 0.5$ at all times.

The FW-H porous control surface used to perform the integration of Equation (13) has been constructed by expanding the wing section offsets by $0.065 \mathrm{~m}$. This distance was chosen so as to avoid any cavities impinging directly onto the surface. Similarly, the downstream extent of the integration surface was chosen to be $0.182 \mathrm{~m}$ downstream of the trailing edge. The exact placement of the control surface with respect to the wing is depicted in Figure 4. Further work is reported on the influence of location of the control surface in Lidtke et al. (2015).

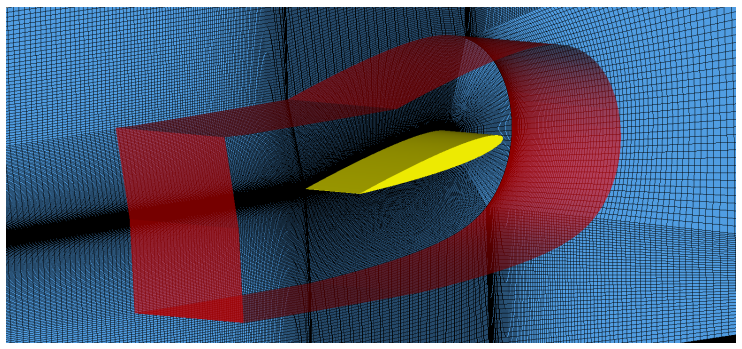

Figure 4: Mesh structure close to the wing (yellow) and the placement of the FW-H integration surface (red).

The inlet was prescribed a fixed velocity of $6.97 \mathrm{~ms}^{-1}$ and the simulation was carried out at the cavitation number of 1.07 which was achieved by using a fixed value of pressure at the outlet of $29 \mathrm{kPa}$ (Foeth et al. 2006). Top and bottom of the numerical cavitation tunnel were treated as slip walls and cyclic boundary condition was prescribed to the span-wise boundaries. Convective outlet velocity condition was used in order to limit the amount of reflections being propagated into the domain for the LES simulations. The wing was treated as a non-slip surface and wall functions were used in order to limit the cell count required in the boundary layer region, following the approach outlined by $\mathrm{Lu}$ et al. (2010). In order to promote convergence from the early stages of the simulations the runs were initialized from a steady-state, non-cavitating flow solution.

Temporal discretisation has been achieved by the use 
of a second-order implicit scheme. For a grid of cell size $\Delta x$ this implied the maximum Courant number limit of $U \Delta x / \Delta t \leq 0.5$ was needed to maintain stability for LES simulations. The volume fraction was discretised using the van Leer scheme with interface compression and a hybrid convection scheme was adopted in which upwinding is applied when required to maintain stability (Lloyd, 2013).

The domain was spatially discretised using a $480 \times 284 \times 40$ grid with 5.4 million elements, most of which were concentrated in and near the boundary layer of the foil and between the wing and the FW-H integration surface. Special care was taken to ensure that the cavities present would not experience rapid changes in mesh density as they are formed, shed and convected downstream of the foil. Similarly, it was ensured that any flow disturbance moving towards the FW-H control surface would not be affected by dissipation errors associated with large changes in mesh topology. The mesh was created using a set of in-house Python libraries combined with the OpenFOAM blockMesh utility. The first wall-normal cell height was ensured to fall within $y^{+} \in<30,50>$, and, to achieve appropriate span- and chord-wise resolution of the flow, the mesh was designed to be characterised by $x^{+}<200$ and $z^{+}<350$. The grid had been subject to a convergence study whereby the relative change in the predicted steady-state, non-cavitating force coefficients was investigated and found to be less than $2 \%$ when compared to a mesh with 9.0 million cells.

\subsection{Results and Discussion}

Several receivers were placed around the wing in order to record the predicted noise pressure levels. These were located approximately $50 \mathrm{~m}$ from the foil and their detailed locations are described in Table 2.

Table 2: Locations of receivers used to predict noise around the hydrofoil.

\begin{tabular}{cccc}
\hline Receiver & $\mathbf{x}[\mathbf{m}]$ & $\mathbf{y}[\mathbf{m}]$ & $\mathbf{z}[\mathbf{m}]$ \\
\hline 10.00 & 50.00 & $s / 2$ & \\
250.00 & 0.00 & $s / 2$ & \\
30.00 & -50.00 & $s / 2$ & \\
$4-50.00$ & 0.00 & $s / 2$ &
\end{tabular}

In order to allow correlation between the recorded noise levels and the predicted flow features the total vol- ume of the cavity,

$$
V=\sum_{i=0}^{N \text { cells }} \frac{\min \left(0, \alpha_{\text {threshold }}-\alpha\right)}{\left|\alpha_{\text {threshold }}-\alpha\right|}(1.0-\alpha) \text { Vcell }_{i},
$$

was also recorded, together with its extents.

It is important to first understand the flow regime around the hydrofoil, which will dictate how noise is generated. Figure 5 shows the predicted cavity volumes in the entire domain. One may note how for high volume fraction threshold values the signals are relatively smooth and periodic with a dominant frequency of about $20 \mathrm{~Hz}$. This may be surprising as one could expect the actions of the cavity sheet and shed clouds to be distinguishable. However, a more in depth analysis of the flow reveals that a new cavity sheet starts to form before a shed cloud becomes disintegrated, as shown in Figure 6. This implies that the maximum volume of the cavity is reached while a cloud is still present in the vicinity of the foil. It thus becomes apparent that, unless the total cavity volume may be broken down into the contribution of the sheet and the clouds, their action on the radiated noise and other flow parameters may not be distinguished reliably.

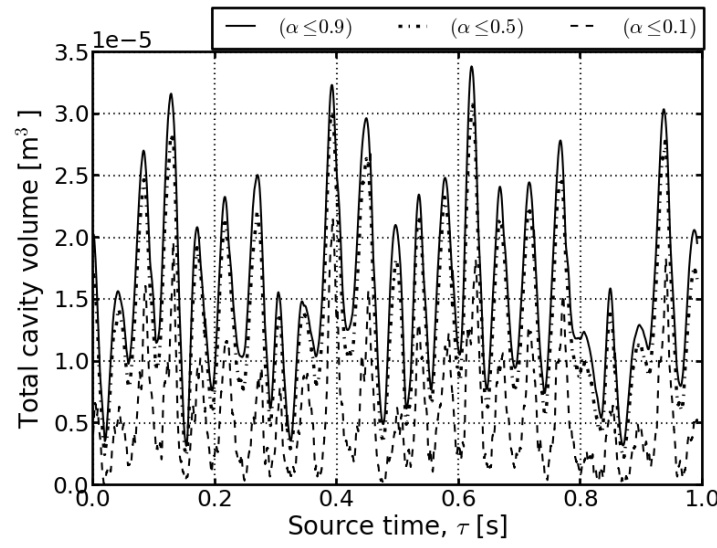

Figure 5: Time history of the total cavity volume for different volume fraction thresholds.

Furthermore, it is also interesting to closely examine the state of the flow at various emission times. Around $\tau=0.125 s$ a large cloud may be seen to shear off from the downstream edge of the cavity sheet, as shown in Figure 7. This is also accompanied by a collapse of a smaller cavity structure, which is trapped in a vortex with its core parallel to and downstream of the trailing edge of the foil.

Next, for time $\tau=0.175 s$ a re-entrant jet may be seen to impact upon the upstream end of the cavity and 


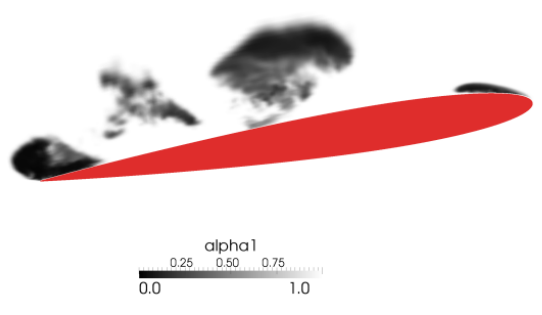

Figure 6: Snapshot of the flow showing formation of a new cavity sheet and the presence of a shed cloud (volume fraction, $\alpha<1.0$ ) downstream of the leading edge.

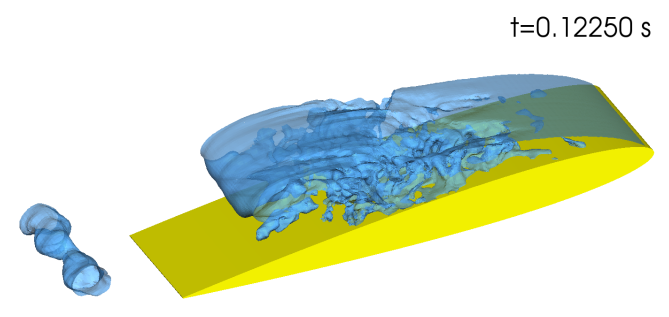

(a)

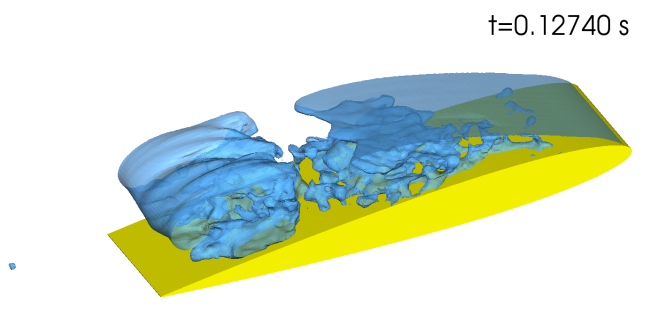

(b)

Figure 7: Shedding of a cavity cloud from the downstream end of the sheet and destruction of a convected cloud trapped in a span-wise vortex. cause a cloud to be shed, as depicted in Figure 8. This is a typical mechanism by which sheet cavitation experiences instability and transient behaviour ( $\mathrm{Lu}$ et al. 2010). There is also a cloud present in the wake of the foil which may be seen to be shrinking rapidly between the two consecutive time steps.

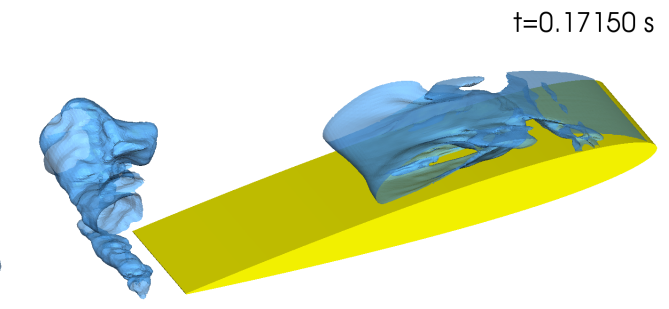

(a)

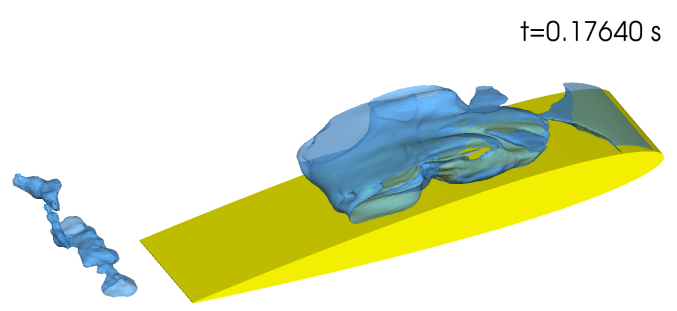

(b)

Figure 8: Necking of the cavity sheet close to the leading edge leading to the shedding of a cloud in a threedimensional manner.

Finally, for the time of $\tau=0.185 s$ the collapse of a shed cloud following the formation of a re-entrant jet is shown in Figure 9. While similar to the already discussed behaviour this event shows a significant amount of three-dimensional disttortion. It is also interesting to note the visible wake of the jet around the mid-span of the wing and how it causes the cavity to fold over the foil from the sides. The later of the associated snapshots also reveals a complicated cavity structure that this event gives rise to.

Results presented in Figure 5 also indicate that there is a significant amount of small-scale oscillations of the cavity interface, particularly for well-defined cavities with low bounding volume fraction thresholds. These are likely to interact strongly with local disturbances to the flow, both creating and being affected by turbulence. This becomes more apparent as one examines the velocity distribution in the wake of the foil, shown in Figure 10. It is also worth noting how the flow circumnavigates 


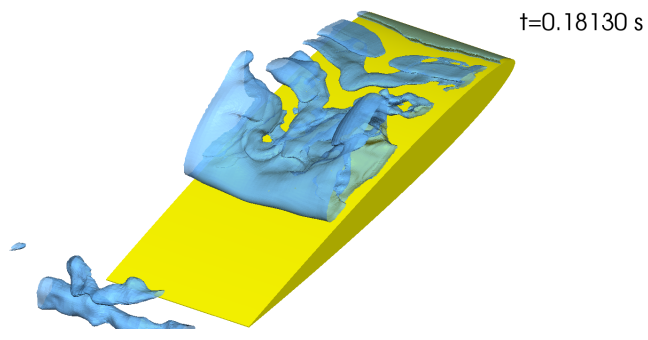

(a)

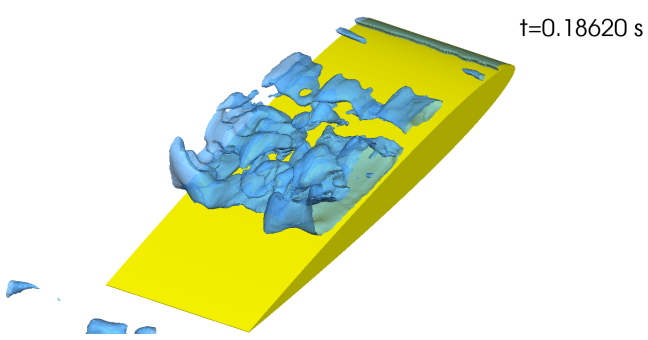

(b)

Figure 9: Complex three-dimensional cavity cloud collapse event following the passing of the re-entrant jet.

the cavities, showing how they act to effectively modify the wing profile shape. The turbulent wake is also likely to affect the noise signature.

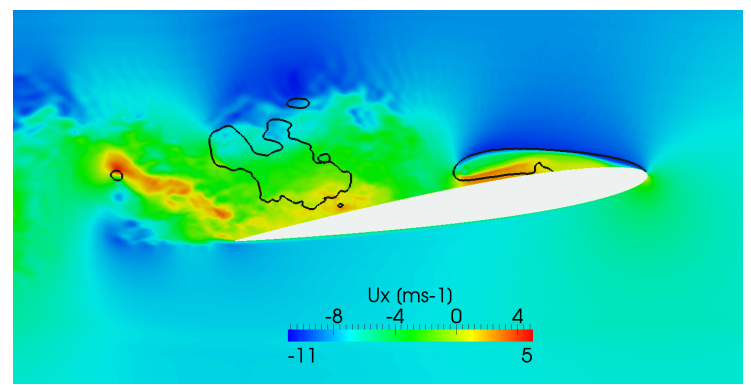

Figure 10: Span-normal cut showing instantaneous axial velocity and volume fraction field iso-contour, $\alpha=$ 0.9 (black), at $z / s=0.5$ and simulation time $\tau=$ $0.160 s$.

Finally, it is worth examining the sound pressure levels predicted at receivers placed around the hydrofoil, presented in Figure 11. One may note that all of them see nearly identical spectral characteristics despite their different positions. The dominant frequency in the signals also coincides closely with that observed for the cavity volume fluctuations, namely $22 \mathrm{~Hz}$. This indi- cates direct dependence of the far-field radiated sound on the oscillation of the cavity volumes and indicates that hte noise source is of monopole nature, as expected from the literature.

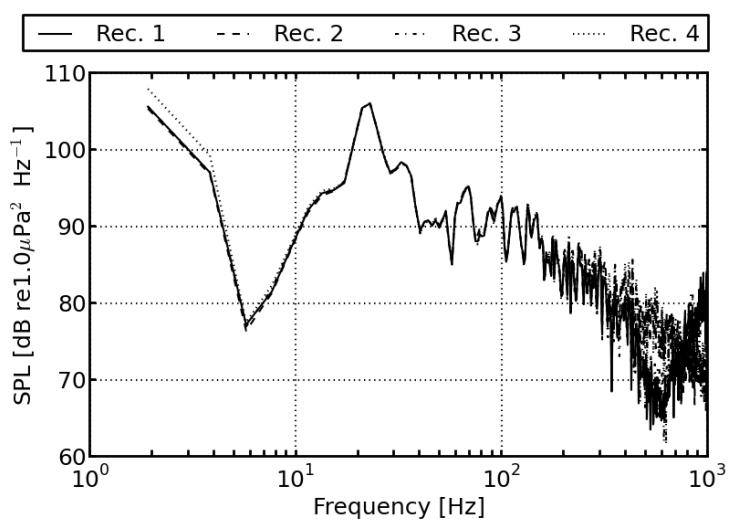

Figure 11: Predicted sound pressure levels for receivers placed in a circumferential manner around the hydrofoil.

\section{Conclusions}

It has been shown that appreciably accurate prediction of the cavitation extents may be made for a propeller at a typical loading condition using unsteady RANS combined with a mass transfer cavitation model. This is of particular importance to the designers, who may utilise such cost-effective computational methods in order to support their decision making process. Drawbacks of this method are, however, the inability to resolve the vortical and other turbulent structures accurately without significant mesh refinement. Blade pass tonal noise has also been predicted and showed expected behaviour. Due to the cavities being relatively stable in the present case no associated noise source could be predicted. It should also be noted that RANS methods are known for their inability to accurately account for cavitation dynamics. Thus, while it may still be possible to gain substantial insight into the nature of the noise generation mechanisms with said approach for initial design, it is likely that detailed design will require the increase in computational costs associated with Large Eddy Simulation and similar approaches. These offer the potential benefit of substantially increasing the accuracy of the unsteady flow predictions.

The second of the presented studies, focused on the noise analysis of a hydrofoil, has indicated that detailed 
insight may be gained into the nature of the noise generation mechanisms when high-fidelity turbulence modelling is employed. In particular, the analysis of the presented data has showed that the noise signature of dominant events such as cloud shedding and sheet formation may be captured. These have been shown to be the primary noise sources. Because an incompressible flow formulation is used it is unlikely that the model used predicts these final stages of oscillation of cavity volume accurately. This, in turn, implies that the input data to the acoustic analogy becomes corrupted, making its predictions incorrect. Studies involving compressible flow simulations, such as those by Wang \& Ostoja-Starzewski (2007) and Seo et al. (2008), have reported that far more physical results may be obtained if the incompressible assumption is not invoked. However, undertaking compressible simulations of marine problems is still an uncommon practice and, reportedly, may be associated with significantly increased computational costs (Wikstrom 2006, Godderidge et al. 2009, Budich et al. 2015, Fujiyama 2015).

Furthermore, it has been discussed, for instance by Wang \& Brennen (1995), that complex collapse mechanisms may occur during collapse of cloud cavities. Predicting these, reportedly, requires one to consider the behaviour of individual bubbles more carefully than what may be achieved using a volume of fluid approach, even if it accounts for some of the compressible effects. This limitation becomes more significant in areas of the flow where intermediate volume fractions become observed, as was the case in the present NACA foil simulations where large cavities break up to form clouds of smaller bubbles. Similar observations have also been reported by others for the PPTC case during inclined shaft simulations when mass transfer models were unable to capture bubble cavitation and were instead indicating the presence of continuous sheet cavities (SVA Hydrodynamic Solutions, 2015). On the one hand it indicates the need for using fine numerical grids to keep mesh-induced effects to an absolute minimum. Moreover, it also highlights one of the fundamental disadvantages of utilizing the VOF approach when modelling disperse phases with no well-defined interface.

It may thus be concluded that the proposed numerical approach based on a mass transfer cavitation model and acoustic analogy is readily capable of predicting the low frequency components of the cavitation noise. It suffers, however, from not being able to predict the effect of small bubbles and because the fluid is considered to be incompressible and hence some noise components are expected to be lost from the analysis The above indicate that the discussed method provides a useful tool allow- ing greater insight into the nature of cavitation noise but requires further refinement in order to be more reliable.

\section{Acknowledgements}

The authors would like to acknowledge the use of OpenFOAM libraries and the Iridis 4 supercomputer of the University of Southampton for all of the presented simulations.

\section{References}

Abdel-Maksoud, M. (ed) (2011), Proceedings of the Workshop on Cavitation and Propeller Performance, Second International Symposium on Marine Propulsors - SMP11, 1718 June, Hamburg, Germany

SVA Hydrodynamic Solutions (2015), www.svapotsdam.de/pptc_data_15.html (on-line), last accessed: $15 / 07 / 2015$

Bensow, R., \& Liefvendahl, M. (2008), Implicit and explicit subgrid modeling in les applied to a marine propeller, 38th Fluid Dynamics Conference and Exhibit, pp. 112

Bensow, R. E., \& Bark, G. (2010). Simulating cavitating flows with LES in OpenFOAM, European Conference on Computational Fluid Dynamics, pp. 1417, Lisbon, Portugal.

Bertschneider, H., Bosschers, J., Choi, G. H., Ciappi, E., Farabee, T., Kawakita, C., and Tang, D., (2014), Final Report and Recommendations to the 27th ITTC, Specialist Committee on Hydrodynamic Noise

Budich, B., Schmidt, S. J., and Adams, N. A., (2015), Numerical Investigation of a Cavitating Model Propeller Including Compressible Shock Wave Dynamics, in Fourth International Symposium on Marine Propulsors

Di Francescantonio, P., (1997), 'A new boundary integral formulation for the prediction of sound radiation,' J. Sound Vib., vol. 202, no. 4, pp. 491509

Ffowcs Williams, J. \& Hawkings, D. L. (1969), Sound generation by turbulence and surfaces in arbitrary Motion, Philosophical Transactions of the Royal Society of London Series A, Mathematical and Physical Sciences, vol. 264, no. 1151 , pp. 321342

Foeth, E. J., Doorne, C. W. H., van Terwisga, T., Wieneke, B. (2006), Time resolved PIV and flow visualization of 3D sheet cavitation, Experiments in Fluids, vol. 40, pp. 503513

Fujiyama, K., (2015), Numerical Simulation of Ship Hull Pressure Fluctuation Induced by Cavitation on Propeller with Capturing the Tip Vortex, in Fourth International Symposium on Marine Propulsors 
Godderidge, B., Turnock, S. R., Earl, C., \& Tan, M., 'The effect of fluid compressibility on the simulation of sloshing impacts,' Ocean Eng., vol. 36, no. 8, pp. 578587, 2009.

Hildebrand, J. (2009), Anthropogenic and natural sources of ambient noise in the ocean, Marine Ecology Progress Series, vol. 395, pp. 520

Hsiao, C.-T., \& Chahine, G. (2004), Prediction of tip vortex cavitation inception using coupled spherical and nonspherical bubble models and Navier-Stokes computations, Journal of Marine Science and Technology, vol. 8(3), pp. 99108

Ianniello, S., Muscari, R., and Mascio, A. D. (2012), Hydroacoustic characterization of a marine propeller through the acoustic analogy, Sustainable Maritime Transportation and Exploitation of Sea Resources, pp. 9911000

Ianniello, S., Muscari, R., and Mascio, A., (2013), Ship underwater noise assessment by the acoustic analogy. Part I: nonlinear analysis of a marine propeller in a uniform flow, J. Mar. Sci. Technol., vol. 18, no. 4, pp. 547570

Ianniello, S. and De Bernardis, E., (2015), Farassats formulations in marine propeller hydroacoustics, Int. J. Aeroacoustics, vol. 14, no. 1 \& 2, pp. 87103

Jamaluddin, A. R., Ball, G. J., Turangan, C. K., Leighton, T. G. (2011), The collapse of single bubbles and approximation of the far-field acoustic emissions for cavitation induced by shock wave lithotripsy, Journal of Fluid Mechanics, vol. 677, pp. 305341

Kellet, P., Turan, O., and Incecik, A., (2014), Underwater Noise and Marine Wildlife: Current and Future Implication and Assessment, in A. Ycel Odaba $\AA$ Colloquium Series, pp. 6369.

Kirsteins, I., Clark, P., Atlas, L. (2011), Maximumlikelihood estimation of propeller noise modulation characteristics, Underwater Acoustic Measurements: Technologies and Results

Lidtke, A. K., Turnock, S. R., \& Humphrey, V. F. (2014), The influence of turbulence modelling techniques on the predicted cavitation behaviour on a NACA0009 foil, $\mathrm{Nu}-$ merical Towing Tank Symposium (NuTTS), Marstrand, Sweden.

Lidtke, A. K., Turnock, S. R., \& Humphrey, V. F. (2015), Characterisation of sheet cavity noise of a hydrofoil using the Ffowcs Williams-Hawkings acoustic analogy, submitted to Computers \& Fluids

Lloyd, T. P., Turnock, S. R., \& Humphrey, V. F., (2014), 'Assessing the influence of inflow turbulence on noise and performance of a tidal turbine using large eddy simulations,' Renewable Energy, vol. 71, pp. 742754, 2014.
Lloyd, T.P., Rijpkema, D., and Van Wijngaarden, E., (2015), Marine propeller acoustic modelling : comparing CFD results with an acoustic analogy method, in Fourth International Symposium on Marine Propulsors

Lu, N., Bensow, R. E., Bark, G. (2010), LES of unsteady cavitation on the delft twisted foil, Journal of Hydrodynamics, Ser. B, vo. 22(5), pp. 784791

Lyrintzis, A. S. (2002), Surface Integral Methods in Computational Aeroacoustics - From the CFD NearField to the Acoustic Far-Field, CEAS Workshop From CFD to CAA, (Athens, Greece), pp. 153

Park, C., Seol, H., Kim, K., Seong, W. (2009),A study on propeller noise source localization in a cavitation tunnel, Ocean Engineering, vol. 36, pp. 754762

Phillips, A. B., and Turnock, S. R., (2013), Application of the VORTFIND algorithm for the identification of vortical flow features around complex three-dimensional geometries, International Journal for Numerical Methods in Fluids, Vo. 71(11), pp. 1461-1474

Plesset, M. S. \& Prosperetti, A. (1977), Bubble dynamics and cavitation, Annual Review of Fluid Mechanics

Salvatore, F. \& Ianniello, S. (2002), Preliminary results on acoustic modelling of cavitating propellers, IABEM 2002, International Association for Boundary Element Methods, UT Austin, TX, USA

Salvatore, F. (2009), Propeller cavitation modelling by CFD-Results from the VIRTUE 2008 Rome workshop,Proc. Of 1st international Symposium on Marine Propulsors, (Trondheim, Norway)

Sauer, J. \& Schnerr, G. H. (2001), Development of a new cavitation model based on bubble dynamics, Zeitschrift fur Angewandte Mathematik und Mechanik, vol. 81, pp. 561562

Seol, H., Suh, J.-C., Lee, S. (2005), Development of hybrid method for the prediction of underwater propeller noise, Journal of Sound and Vibration, vol. 288, pp. 345360

Seol, H. (2013), 'Time domain method for the prediction of pressure fluctuation induced by propeller sheet cavitation: Numerical simulations and experimental validation,' Ocean Eng., vol. 72, pp. 287296

Seo, H, Moon, Y. J., and Shin, B. R. (2008), Prediction of cavitating flow noise by direct numerical simulation, J. Comput. Phys., vol. 227, no. 13, pp. 65116531

Tasker, M. L., Amundin, M. et al. (2010), Marine Strategy Framework, Task Group 11 Report: Underwater noise and other forms of energy, European Commission Joint Research Centre, Institute for Environment and Sustainability

Turnock, S.R., Pashias, C. and Rogers, E., (2006), Flow feature identification for capture of propeller tip vortex evolution. In Proceedings of the 26th Symposium on 
Naval Hydrodynamics, Rome, IT, INSEAN Italian Ship Model Basin / Office of Naval Research, pp. 223-240

Urick, R. J. (1984), Ambient Noise in the Sea, Undersea Warfare Technology Office, Naval Sea Systems Command, Dept. of the Navy, Washington D.C.

Vallier, A. (2013), Simulations of cavitation-from the large vapour structures to the small bubble dynamics, $\mathrm{PhD}$ thesis, Lund University

Van der Graaf, A., Ainslie, M. et al. (2012),European Marine Strategy Framework Directive: Good Environmental Status (MSFD-GES), Tech. Rep.

Wang, Y.-C. and Brennen, C. E. (1995), 'the Noise Generated By the Collapse of a Cloud of Cavitation Bubbles,' 1995 ASME/JSME Fluids Eng. Laser Anemometry Conf. Exhib., vol. 226, pp. 1729

Wang, G. \& Ostoja-Starzewski, (2007), Large Eddy Simulation of a sheet/cloud cavitation on a NACA0015 hydrofoil, Applied Mathematical Modelling, vo. 31(3), pp. 417-447

Wikstrom M. (2006), Approaching Large Eddy Simulation of cavitating flows for marine applications, $\mathrm{PhD}$ Thesis, Chalmers University of Technology

Woo Shin, K. (2010), Cavitation simulation on marine propellers, $\mathrm{PhD}$ thesis, Technical University of Denmark 